\title{
Experimental validation of systematically designed acoustic hyperbolic meta material slab exhibiting negative refraction
}

\section{Christiansen, Rasmus Ellebæk; Sigmund, Ole}

\section{Published in:}

Applied Physics Letters

Link to article, DOI:

$10.1063 / 1.4962441$

Publication date:

2016

Document Version

Peer reviewed version

Link back to DTU Orbit

Citation (APA):

Christiansen, R. E., \& Sigmund, O. (2016). Experimental validation of systematically designed acoustic hyperbolic meta material slab exhibiting negative refraction. Applied Physics Letters, 109(10), [101905]. https://doi.org/10.1063/1.4962441

\section{General rights}

Copyright and moral rights for the publications made accessible in the public portal are retained by the authors and/or other copyright owners and it is a condition of accessing publications that users recognise and abide by the legal requirements associated with these rights.

- Users may download and print one copy of any publication from the public portal for the purpose of private study or research.

- You may not further distribute the material or use it for any profit-making activity or commercial gain

- You may freely distribute the URL identifying the publication in the public portal 


\author{
Rasmus E. Christiansen* and Ole Sigmund. \\ Department of Mechanical Engineering, Solid Mechanics, \\ Technical University of Denmark, Nils Koppels Allé, B. 404, \\ DK-2800 Lyngby, Denmark
}

\begin{abstract}
This Letter reports on the experimental validation of a two-dimensional acoustic hyperbolic meta material slab optimized to exhibit negative refractive behavior. The slab was designed using a topology optimization based systematic design method allowing for tailoring the refractive behavior. The experimental results confirm the predicted refractive capability as well as the predicted transmission at an interface. The study simultaneously provides an estimate of the attenuation inside the slab stemming from boundary layer effects - insight which can be utilized in the further design of meta material slabs. The capability of tailoring the refractive behavior opens possibilities for different applications. For instance, a slab exhibiting zero refraction across a wide angular range is capable of funneling acoustic energy through it, while a material exhibiting negative refractive behavior across a wide angular range provides lensing and collimating capabilities.
\end{abstract}

This Letter presents the experimental validation of an acoustic hyperbolic meta material (AHMM) slab obtained using a systematic design method proposed recently in [1]. The negative refraction exhibited by the slab stems from a hyperbolic band effect in the second acoustic band.. Negative refraction is demonstrated in a $10 \%$ frequency band for a localized acoustic wave, incident on the AHMM slab across the angular interval, $\theta_{1} \in\left[0^{\circ}, 25^{\circ}\right]$. Predicted high energy transmittance through the material-air interface is experimentally verified at normal incidence. The index of refraction, $n$, is defined according to Snell's law.

All known naturally occurring materials have positive $n$. Over the last two decades significant effort, initiated by Pendry [2] and the earlier work by Veselago [3], has been devoted to designing meta materials (MMs) exhibiting exotic refractive behavior, e.g. $n \leq 0$, across several areas of physics. This includes electromagnetics, solid mechanics and acoustics, see e.g. [4], [5] and [6].

Having access to MMs with exotic material parameters opens a range of engineering possibilities. These range from exotic applications, like cloaking or beating the diffraction limit [7], to the more "mundane" such as creating energy focusing and funneling devices [8] or flat lenses [9]. AHMMs like the one investigated in this Letter may find use in medical ultra sound, spatial filtering or subwavelength imaging.

A recent paper, [10], investigated quasi-two-dimensional acoustic MMs simultaneously exhibiting effective negative mass density and bulk modulus. Promising theoretical results were presented, whereas experiments showed strong attenuation in the frequency region of doubly negative material parameters, severely limiting the practical usability of the exotic material property. While attenuation is observed for the AHMM slab considered in this Letter, it is smaller and the refractive behavior is clearly demonstrated.

Another recent Letter on the topic of AHMMs exhibiting negative refraction is [8]. Here a 3D AHMM slab, exhibiting

\footnotetext{
* Corresponding email: raelch@ mek.dtu.dk
}

$n \leq 0$ was investigated numerically and experimentally. Frequency dependent negative refraction was demonstrated over $a \approx 5 \%$ frequency band for waves at high angle of incidence, $\theta_{1} \geq 25^{\circ}$ and close to no refraction for $\theta_{1}<25^{\circ}$. Where the AHMM considered in [8] only exhibits negative refraction for high values of $\theta_{1}$ the AHMM slab considered in this Letter is specifically tailored to exhibit negative refraction from $\theta_{1}=0^{\circ}$.

The systematic design method suggested in [1], considers a finite arrangement of identical unit cells inserted in a homogeneous acoustic medium thereby inherently accounting for the coupling between the slab and the external field. Thus the method simultaneously optimizes the slabs towards exhibiting the desired refractive behavior and a high energy transmission. This is in contrast to much earlier work on designing MMs with exotic material parameters which is based on a single periodic unit cell, not taking the finite size of the physical system into account, hereby neglecting the coupling into and out of the MM.

In short, the method considers a $2 \mathrm{D}$ exterior acoustic problem, modeled using the Helmholtz equation in a finite domain $\Omega$, see Fig $1 \mathrm{i}$, with a far field matching condition imposed along $\delta \boldsymbol{\Omega}$. The slab consists of an array of identical axisymmetric unit cells in $\boldsymbol{\Omega}_{\mathrm{d}}$. A localized near-plane wave, $\psi$, impinging on the slab at a prescribed angle of incidence, $\theta_{1}$, and frequency, $f$, is introduced using a Neumann boundary condition at $\delta \Omega_{\mathrm{PW}}$. The design problem is formulated as a PDE-constrained minimization problem with the objective function being the standard deviation between $|\psi|$ and the absolute value of a prescribed wave $\left|\psi_{\text {target }}\right|$ over the subdomain $\boldsymbol{\Omega}_{\mathrm{op}}$. The position of $\psi_{\text {target }}$ selects the desired refraction angle at the slab interface, $\theta_{\text {ref }}$, and by extension the index of refraction, $n_{\text {target }}\left(\theta_{1}, f\right)$. The discrete material distribution problem is replaced by a continuous problem and is solved using density based topology optimization [11].

The AHMM slab studied in this Letter consists of $20 \times$ 9 unit cells of size $2 \mathrm{~cm} \times 2 \mathrm{~cm}$. The unit cell, originally presented in [1], is shown in the top image of Fig. 1ii. The slab was produced in ABS plastic [Stratasys ABS-P430] with 


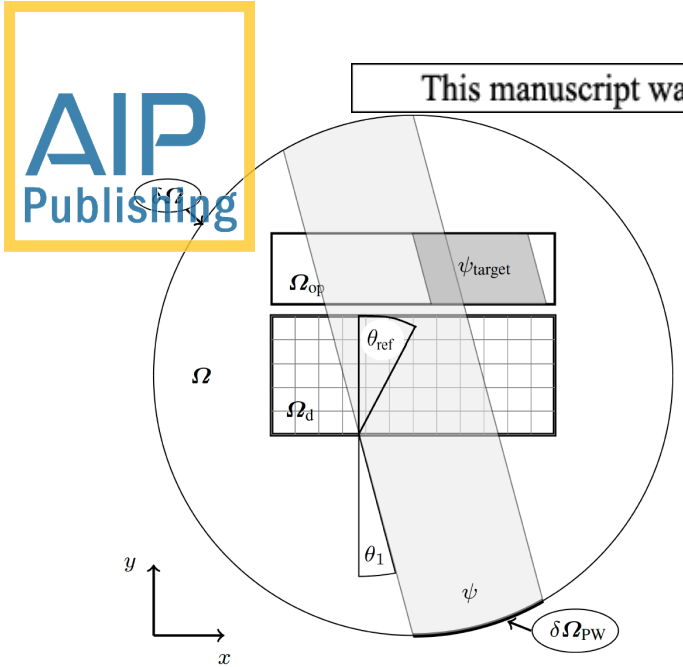

(i)

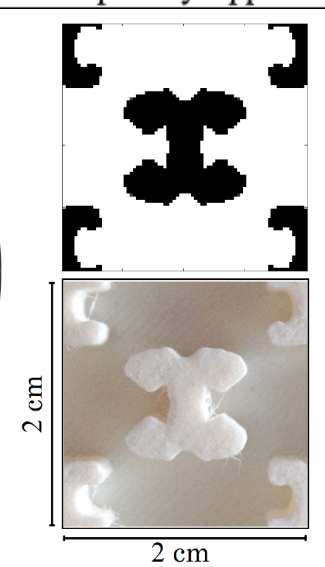

(ii)

FIG. 1: (color online) (i) Sketch of model problem used in the systematic design method. Reproduced with permission from [1]. Copyright 2016 Springer Verlag. (ii) [Top] Designed unit cell, Reproduced with permission from [1]. Copyright 2016 Springer Verlag. [Bottom] 3D-printed unit cell.

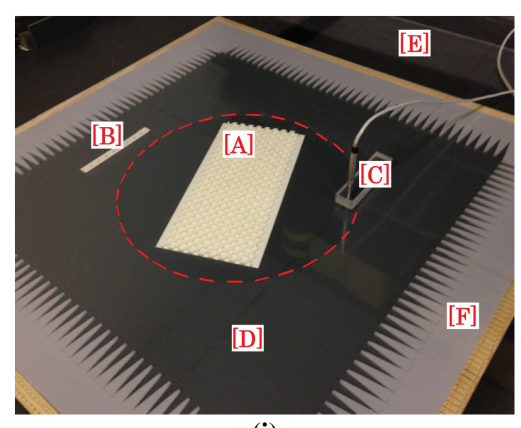

(i)

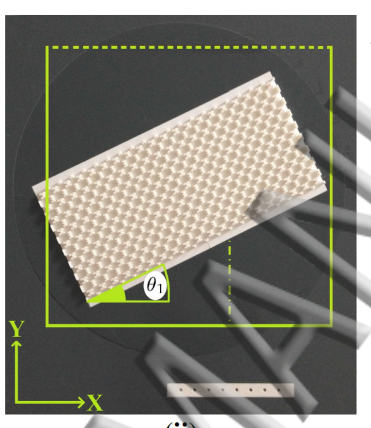

(ii)

FIG. 2: (color online) (i) Experimental setup. [A]: MM slab, [B]: mini-loudspakers array, [C]: Flush mounted microphone, [D]: PVC baseplate, [E] Acrylic top plate, [F] Absorbing foam. The dashed red line indicates the rotatable disk. (ii) Aerial view of experimental setup. The square outlines the measurement area for the data in Fig.

3i. The data in Figs. 4i-iii and Fig. 5iii was obtained along the horizontal dashed line and the dash-dotted vertical line, respectively.

measured values of density, $\rho=9.54 \cdot 10^{2} \mathrm{~kg} / \mathrm{m}^{3}$ and bulk modulus $\kappa=1.9 \mathrm{GPa}$, using 3D-printing (with an accuracy of $0.2 \mathrm{~mm}$ ) as two blocks of $10 \times 9$ unit cells consisting of a $20 \mathrm{~cm} \times 20 \mathrm{~cm}$ baseplate of $1 \mathrm{~cm}$ thickness with the slab printed on top to a height of $0.6 \mathrm{~cm}$. A 3D-printed unit cell is shown in the bottom image of Fig. 1ii. A result of the design and manufacturing process is that the unit cells along the edge of the MM slab have a slightly altered geometry, effectively removing the thin horizontal features at the top and bottom in the top image of Fig. 1ii.

The experimental setup, building on the approach in [12], is shown in Fig. 2i. A $110 \mathrm{~cm} \times 110 \mathrm{~cm}, 1 \mathrm{~cm}$ thick PVC plate served as a baseplate, [D]. A circular hole of radius $25 \mathrm{~cm}$ was cut in its center. A $1 \mathrm{~cm}$ thick freely rotatable PVC disk of (near) identical radius ( $0.5 \mathrm{~mm}$ accuracy) was placed into the hole. A rectangular hole of $40 \mathrm{~cm} \times 20 \mathrm{~cm}$

was cut in the disk and the AHMM slab inserted, [A]. A rectangular hole of $19 \mathrm{~cm} \times 2 \mathrm{~cm}$ was cut in the baseplate used to mount a line-array of 8 mini-loudspeakers, [B]. Wooden strips of $0.6 \mathrm{~cm}$ height were fixed along the baseplate edges. $13 \mathrm{~cm}$ wide blocks of open cell absorbing foam made from melamine resin [Basotec from BASF] of 0.6 $\mathrm{cm}$ height, $[\mathrm{F}]$, were attached along the strips. $6.5 \mathrm{~cm}$ deep, $1.5 \mathrm{~cm}$ wide wedges were cut into the blocks at a $2 \mathrm{~cm}$ interval for increased attenuation. A freely movable solid $150 \mathrm{~cm} \times 150 \mathrm{~cm}, 1 \mathrm{~cm}$ thick transparent acrylic plate, [E], was placed on top of the wooden strips, forming a closed cavity. The cavity height assures an essentially two dimensional pressure field for $f<f_{\text {cut }} \approx 28.5 \mathrm{kHz}$. An $1 / 8$ 'th inch microphone (Brüel and Kjær, Nærum Denmark) was flush mounted in the acrylic plate, [C]. The microphone was attached to a computer through a NEXUS pre-amplifier and a PULSE analyzer (Brüel and Kjær, Nærum Denmark). The loudspeakers were connected to a tone generator [Type 1049 (Brüel and Kjær, Nærum Denmark)] through a RME sound card [Hammerfall DSP Multiface II]. The output of each loudspeaker, $\psi_{i}, i \in\{1,2, . ., 8\}$, was calibrated to a level of $(75 \pm 0.1) \mathrm{dB}$ SPL ref $20 \mu \mathrm{Pa}$ measured in the empty cavity at $7 \mathrm{~cm}$ distance. The output was then modulated from left to right as: $\psi_{i}=\psi_{i}+\Delta \psi_{i}, \Delta \psi_{i} \in$ $\{-13.5,-7.0,-2.0,0.0,0.0,-2.0,-7.0,-13.5\} \mathrm{dB}$ SPL ref $20 \mu \mathrm{Pa}$. In this configuration the loudspeakers emit a localized approximate plane wave front perpendicular to the array. Fig. 2ii shows an aerial view of the setup, including markings of different measurement areas for later reference.

The angle of incidence, $\theta_{1}$, of the pressure wave onto the slab was adjusted by rotating the disk (with uncertainty in $\theta_{1}$ of $\leq \pm 0.25^{\circ}$ ). The pressure field was recorded by translating the acrylic plate, hereby scanning the flush mounted microphone across the cavity. An average of 20 measurements was used for each position (with uncertainty in position $\leq \pm 1 \mathrm{~mm}$ ). The setup allows for measuring the field inside the slab, by aligning the microphone with void regions in the slab.

First, the pressure field for $\theta_{1}=14^{\circ}$ and $f=8575 \mathrm{~Hz}$ is considered. Outside the slab the pressure was measured in a Cartesian grid with $1 \mathrm{~cm}$ spacing for $x \in[-20 \mathrm{~cm}, 22 \mathrm{~cm}]$ and $y \in[-20 \mathrm{~cm}, 20 \mathrm{~cm}]$. Inside the slab it was measured in a staggered grid with two grid points per unit cell. A contour plot of the measured field is shown in Fig. 3i. The black square shows the position of the slab. The black dashed line denotes the direction with no refraction and the gray line denotes the direction of $n=-1$. White regions correspond to missing data. A simulation was performed in COMSOL 5.2 using a $2 \mathrm{D}$ setup mimicking the experimental setup. A line-array of eight point-like sources (modulated as in the experimental setup) was used to generate the pressure. Absorbing material was introduced along the domain edges. An acoustic model was used in the cavity and the Johnson-Champoux-Allard poroacoustic model was used in the absorbing material with the material parameters for the melamine foam sample number 33 in [13]. A contour plot of the simulated pressure field, sampled similarly to the measurements, is presented in Fig. 3ii. Good 


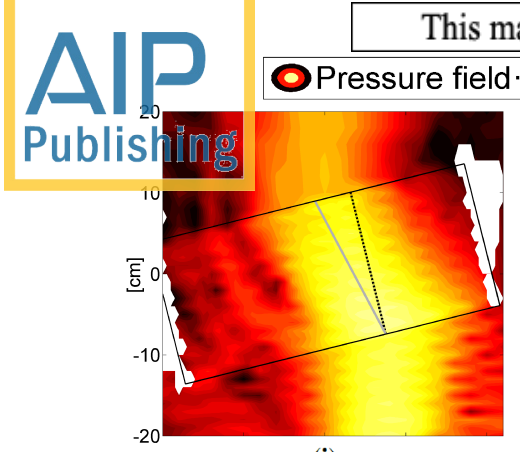

(i)

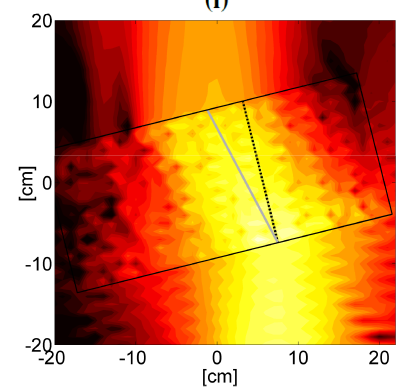

(iii)
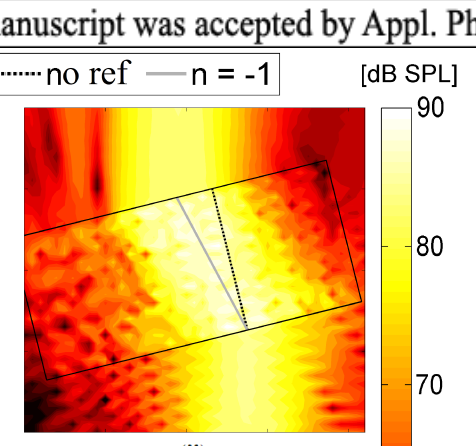

(ii)

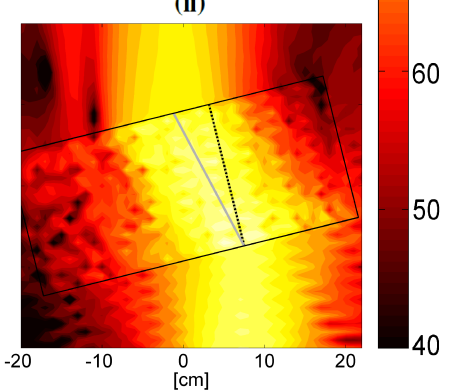

(iv)

FIG. 3: (color online) Pressure field (dB SPL ref $20 \mu \mathrm{Pa}$ ) in the area outlined by the square in Fig. 2ii, with $\theta_{1}=14^{\circ}$ and $f=8575 \mathrm{~Hz}$.

(i) Measured field. (ii) Simulated field (no attenuation). (iii)

Simulated field (attenuation in cavity and slab). (iv) Simulated field (attenuation in cavity). Black square: Position of the slab. The black and gray lines indicate the direction of no refraction and the direction with $n=-1$, respectively.

agreement is observed between the two plots, the greatest discrepancy being the attenuation observed in the measured field. Another small discrepancy is a slight shift in position of the pressure field behind the slab. The physical reasons for the attenuation is discussed below. To estimate the magnitude of the attenuation a reference measurement of the pressure field inside the empty cavity was performed and a value of $0.4 \mathrm{~dB}$ per wavelength identified. The result of a simulation including this estimated attenuation is presented in Fig. 3iv. This result show an improved agreement with the measured result. An estimate of the attenuation in the slab was made by fitting simulated data to the measured field in Fig. $3 \mathrm{i}$ in the area $x \in[-20 \mathrm{~cm}, 22 \mathrm{~cm}], y \in[12 \mathrm{~cm}, 20 \mathrm{~cm}]$. Additional attenuation of $\approx 0.56 \mathrm{~dB}$ per free field wavelength was identified inside the slab. The result of a simulation using the estimated attenuation inside the slab is presented in Fig. 3iii. The agreement between the field in $3 \mathrm{i}$ and 3iii is now remarkable in terms of the pressure level and distribution.

The experimentally observed attenuation inside the chamber and slab can be explained by viscous and thermal boundary effects at surfaces. Based on a model for a circular duct with a diameter of $6 \mathrm{~mm}$ an attenuation of $\approx 0.32 \mathrm{~dB}$ per wavelength is calculated (see chap. 7.7.1 of [14]). This is close to the experimentally estimated $0.4 \mathrm{~dB}$ per wavelength in the cavity. Considering that the unit cell making up the slab contains narrow passages ( $\approx 3 \mathrm{~mm}$ wide), increasing the influence of the boundary effects, provides an explanation of

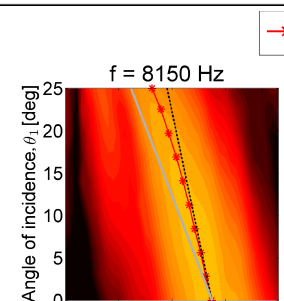

(i)

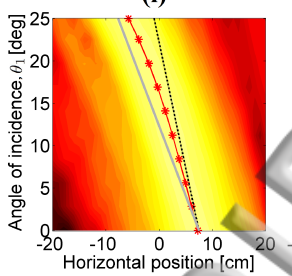

(iv) $\rightarrow-n_{e} \ldots-\cdots \cdot$ no ref $-\mathrm{n}=-1$

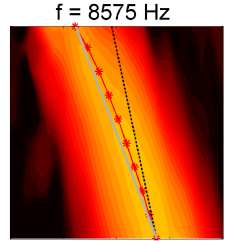

(ii)

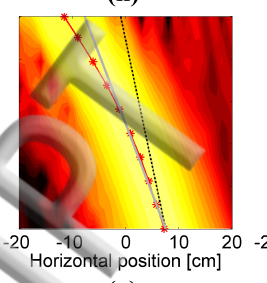

(v)

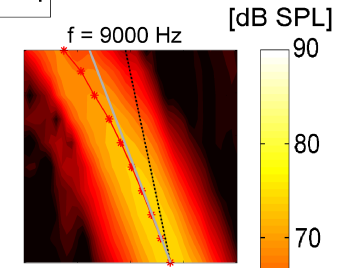

(iii)

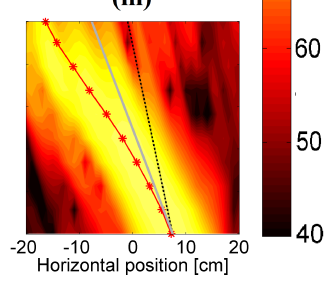

(vi)
FIG. 4: (color online) Pressure field (dB SPL ref $20 \mu \mathrm{Pa}$ ) along the horizontal dashed line in Fig. 2ii as a function of $\theta_{1}$. Black line: direction of no refraction. Gray line: direction with $n=-1$. Red line with asterisks: The position of the center of a Gaussian envelope fitted to the pressure data. (i)-(iii) Measured pressure. (iv)-(vi) Simulated pressure (no attenuation).

the additional attenuation observed inside the slab.

Next the field transmitted through the slab is considered, to investigate its refractive properties as a function of $f$ and $\theta_{1}$. Measurements were performed along the line $x \in$ $[-20 \mathrm{~cm}, 20 \mathrm{~cm}]$ at a regular spacing of $1 \mathrm{~cm}$ at $y=20 \mathrm{~cm}$ (the dashed line in Fig. 2ii). The frequencies, $f \in$ $\{8150 \mathrm{~Hz}, 8575 \mathrm{~Hz}, 9000 \mathrm{~Hz}\}$ and the angular interval $\theta_{1} \in$ $\left[0^{\circ}, 25^{\circ}\right]$ were considered. Fig. 4 shows contour plots of the pressure as a function of horizontal position and $\theta_{1}$. The black and gray lines correspond to no refraction and $n=$ -1 , respectively. The red line, with asterisks marking the measured angles, corresponds to the center of a Gaussian envelope fitted to the pressure data at each $\theta_{1}$-value used to provide a consistent estimate of the refractive index. The first row of Fig. 4 presents measured data while the second row presents simulated data obtained using the COMSOL 5.2 model without attenuation. Negative refraction is seen for all $f$ and $\theta_{1}$ in both the simulated and experimental data. While the pressure field is clearly transmitted through the slab across the full angular interval the field strength is seen to drop at high values of $\theta_{1}$. In addition to the experimental and simulation results Equal Frequency Surface (EFS) analysis is used to estimate $\theta_{\text {ref }}\left(f, \theta_{1}\right)$ as illustrated on Fig. 5ii. Fig. 5i shows the angle of refraction, $\theta_{\text {ref }}$ (see Fig. 1i), at the air to slab interface, calculated from the red line in Fig. 4, as a function of $\theta_{1}$ for the six cases in Fig. 4 along with the three EFS calculations. Simulated data is plotted with unmarked solid curves, measured data with dashed curves and circles denoting the measured angles and the EFS result with solid curves and crosses. In all cases the AHMM slab is observed to exhibit negative refraction across the full angular interval. A consistent increase in $\theta_{\text {ref }}$ is observed from the simulated to the measured data for all $f$. It is believed that the shift in $\theta_{\text {ref }}$ 


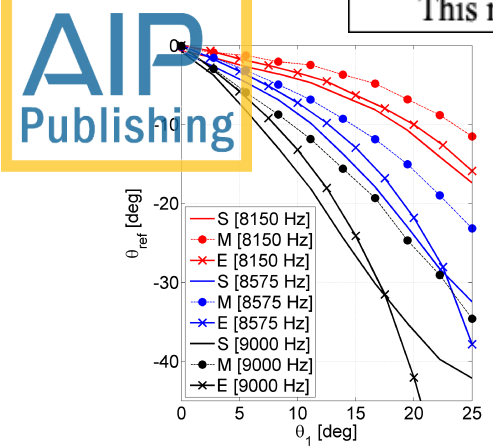

(i)

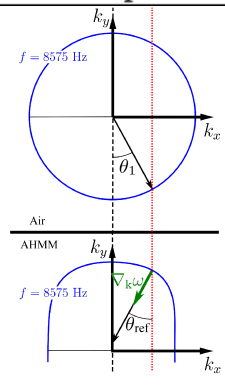

(ii)

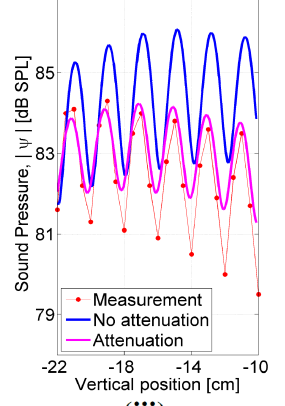

(iii)
FIG. 5: (color online) (i) $\theta_{\text {ref }}$, as a function of $\theta_{1}$. Unmarked full curves correspond to the simulated data in the bottom row of Fig. 4. Dashed curves with circles correspond to the measured data in the top row of Fig 4. Full curves with crosses correspond to the EFS analysis. (ii) Illustration of EFS analysis for determining $\theta_{\text {ref }}\left(f, \theta_{1}\right)$ at air to AHMM interface. (iii) Pressure along the vertical dash dotted line in Fig. 2ii at $\theta_{1}=0^{\circ}$ and $f=8575 \mathrm{~Hz}$.

can be explained by a combination of the following effects. Firstly, the effect of going from the 2D numerical model to the quasi 2D experimental setup. Secondly, a shift in the speed of sound, $c$, caused by differences in operating conditions, e.g. temperature, between the experimental and numerical setups. Thirdly, the aforementioned boundary effects can cause a shift of the phase speed, $c_{p}$, inside the cavity, compared to the free field speed of sound $c$ (see an analysis for a circular duct in chapter 7.7 of [14]). The EFS results agree qualitatively with the simulation results, but predict a higher value for $\theta_{\text {ref }}$ at high values of $\theta_{1}$ for $f \in\{8575 \mathrm{~Hz}, 9000 \mathrm{~Hz}\}$. The small discrepancies are attributed to differences between the finite slab and the infinite EFS case.

Finally, the acoustic energy transmission at the air to slab interface at normal incidence is considered. The pressure field at $f=8575 \mathrm{~Hz}$ and $\theta_{1}=0^{\circ}$ was measured for $y \in[-22 \mathrm{~cm},-10 \mathrm{~cm}], x=7.5 \mathrm{~cm}$ (along the dash dotted line in Fig. 2ii) at a $0.5 \mathrm{~cm}$ regular spacing. The measured field is shown in Fig. 5 iii (red line with asterisks). Two other graphs obtained from simulation data are included. These are: the pressure obtained from the COMSOL 5.2 model without attenuation (blue line) and with attenuation in the cavity and slab (magenta line). The magnitude of the reflection factor, $|R|$, at a material interface at normal incidence, may be calculated from the interference pattern in the reflected field using the peak ratio $s=\min |\psi| / \max |\psi|$, as $|R|=$ $(1-s) /(1+s) .|R|$ is used to determine the transmittance, $\tau$, through the interface as $\tau=1-|R|^{2}$, see chapter 3.1 of [14].

$|R|^{2}$ and $\tau$ for the slab at $f=8575 \mathrm{~Hz}$ and $\theta_{1}=0^{\circ}$ are estimated using the mean value (in Pascal) of $\max |\psi|$ and min $|\psi|$ obtained from the six maxima and five intermediate minima for the data series in Fig. 5iii, see Table I.
Agreement in $\tau$, to within few percent, is observed when comparing simulation with measurement. The measured value of $\tau$ is smaller than the value which can be calculated from the data in [1] for the idealized model problem for which the AHMM was designed $\left(\tau_{\text {ideal }} \approx 0.985\right)$. The deviation may be explained by limitations in the experimental setup. First,

\begin{tabular}{lcc} 
Name: & Reflectance: $|R|^{2}$ & Transmittance: $\tau$ \\
\hline Measurement: & $\approx 0.027$ & $\approx 0.973$ \\
No attenuation: & $\approx 0.035$ & $\approx 0.965$ \\
Attenuation: & $\approx 0.014$ & $\approx 0.986$ \\
\hline
\end{tabular}

TABLE I: Reflectance, $|R|^{2}$, and transmittance, $\tau$, for the air to AHMM interface, obtained from the data in Fig. 5iii.

eight point-like sources do not perfectly model a plane wave front, leading to interference in the pressure field. Second, the slab was designed using a different number of layers, and a change in slab thickness can be expected to introduce Fabry Pérot like variations in the transmittance.

In summary, an AHMM slab obtained using the systematic design method proposed in [1] was investigated experimentally. When accounting for attenuation, a remarkable agreement was observed between simulated and measured data. Even without attenuation, good qualitatively agreement was shown, both with respect to the refractive behavior of the slab and the interface transmission, validated at normal incidence. The presence of thermal and viscous boundary layers at the cavity and slab surfaces is believed to be responsible for the observed attenuation. Negative refraction was demonstrated across a full $10 \%$ frequency band for $\theta_{1} \in\left[0^{\circ}, 25^{\circ}\right]$. The experimentally observed refractive index was consistently shifted compared to the simulated results, a phenomenon also observed for the AHMM considered in [8].

\section{ACKNOWLEDGMENTS}

Assistant Engineers Jørgen Rasmussen and Tom Arent Petersen at the Department of Electrical Engineering at the Technical University of Denmark assisted in preparing the experiments. The workshop at the Department of Mechanical Engineering at the Technical University of Denmark aided in the processing of components for the experimental setup. The 3D-printing laboratory FabLab at the Tecnical University of Denmark produced the test specimen. The work was financially supported by Villum Fonden through the research project Topology Optimization - the Next Generation (NextTop).
[1] R. E. Christiansen and O. Sigmund, Structural and Multidiciplinary Optimization 54, 469?482 (2016).
[2] J. B. Pendry, Physical Review Letters 85, 3966 (2000).

[3] V. G. Veselago, Soviet Physics Uspekhi 10, 509 (1968). 
11. Z Z hang and Z. Liu, Applied Physics Letters 85, 341 (2004). 5] S. Z ang, W. Fan, N. C. Panoiu, K. J. Malloy, R. M. Osgood, Publishilings. R. J. Brueck, Physical Review Letters 95, 137404 (2005). [6] F. D. Philippe, T. W. Murray, and C. Prada, SCIENTIFIC REPORTS 5, 11112 (2015).

[7] R. V. Craster and S. Guenneau, Acoustic Metamaterials Negative Refraction, Imaging, Lensing and Cloaking, edited by R. Hull, C. Jagadish, J. R. M. Osgood, J. Parisi, and Z. M. Wang (Springer Science+Business Media Dordrecht, 2013).

[8] V. M. Garciá-Chocano, J. Christensen, and J. Sánchez-Dehesa, Physical Review Letters 112, 1 (2014).
[9] P. V. Parimi, W. T. Lu, P. Vodo, and S. Sridhar, Nature 426, 404 (2003).

[10] R. Graciá-Salgado, V. M. Garciá-Chocano, D. Torrent, and J. Sánchez-Dehesa, Physical Review B 88, 1 (2013).

[11] M. P. Bendsøe and O. Sigmund, Topology Optimization (Springer, 2003).

[12] R. E. Christiansen, E. Fernandez-Grande, and O. Sigmund, Journal of the Acoustical Society of America 138 (6), 3470 (2015).

[13] N. Kino and T. Ueno, Applied Acoustics 69, 325 (2008).

[14] F. Jacobsen and P. M. Juhl, Fundamentals of General Linear Acoustics (WILEY, 2013). 


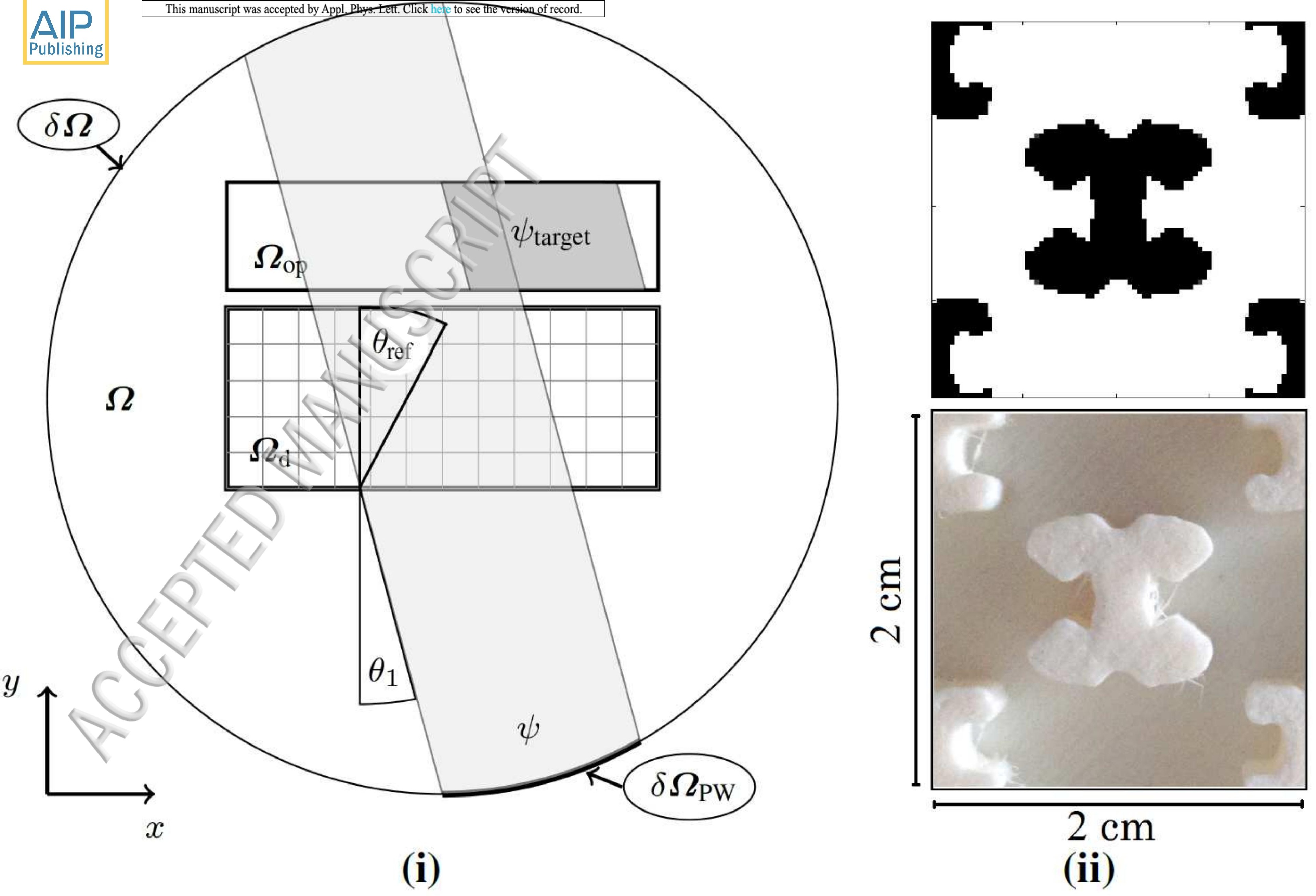




\section{$\rightarrow * \mathrm{n}_{\mathrm{e}} \ldots \cdots$ no ref $-\mathrm{n}=-1$}

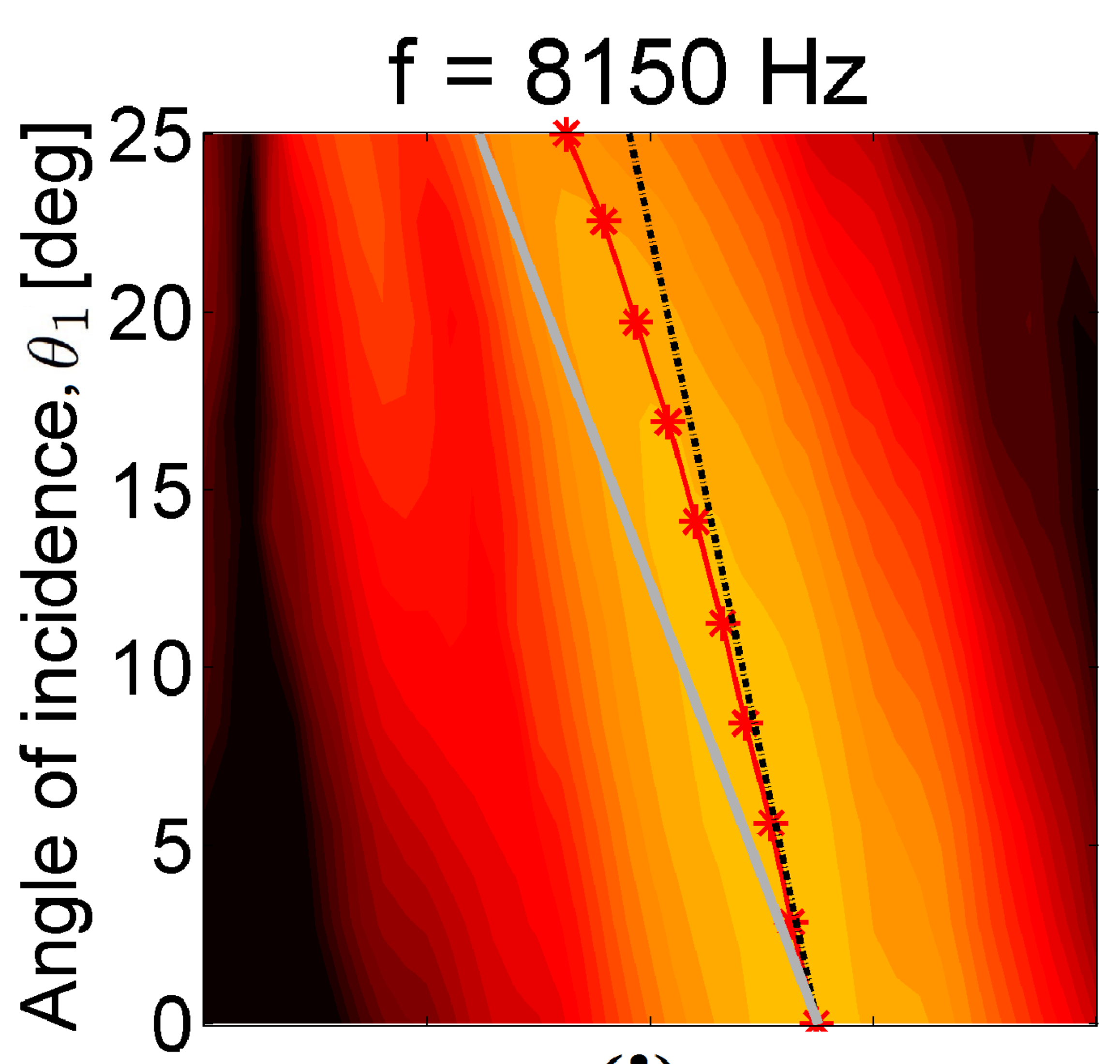

(i)

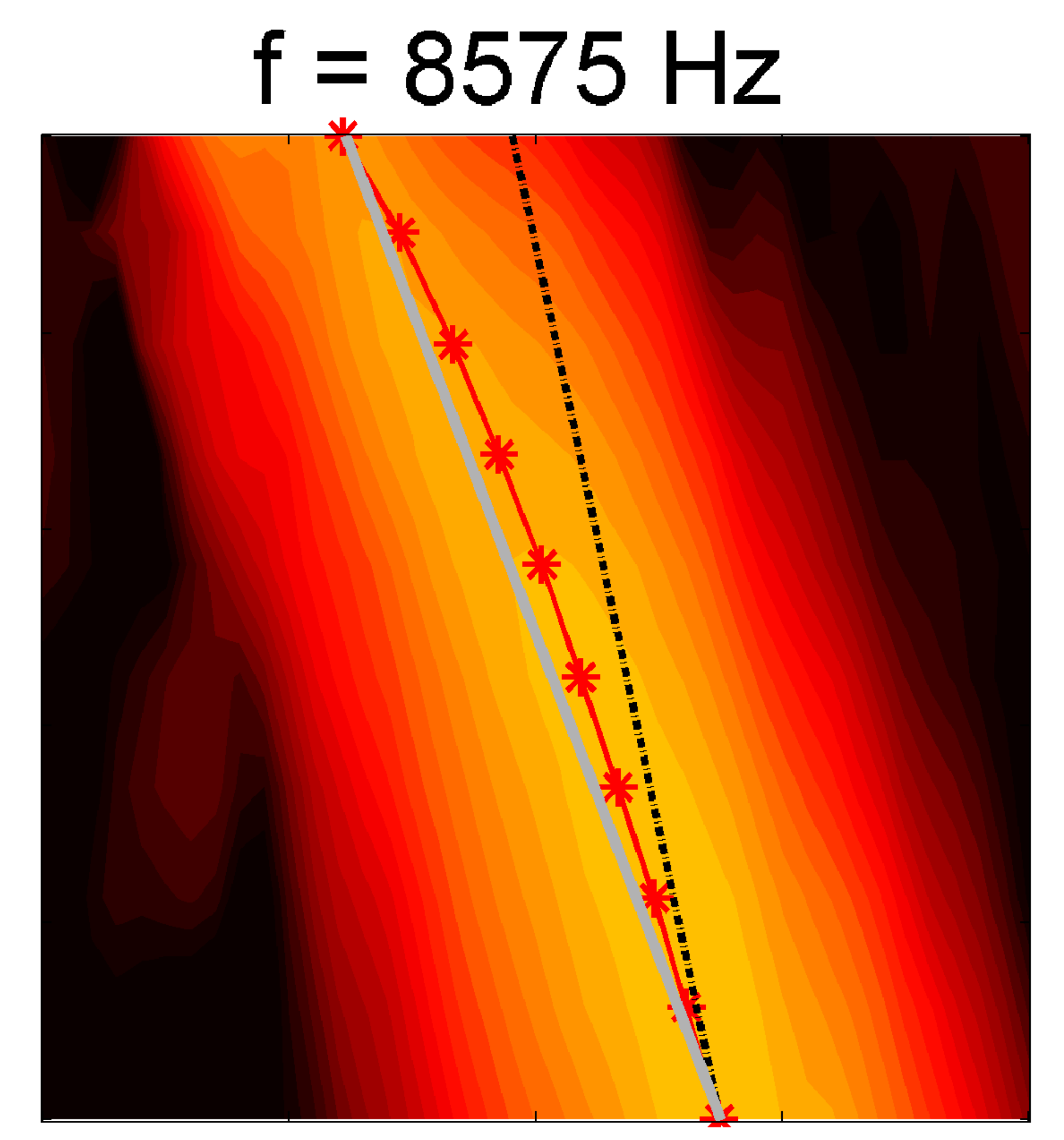

(ii)

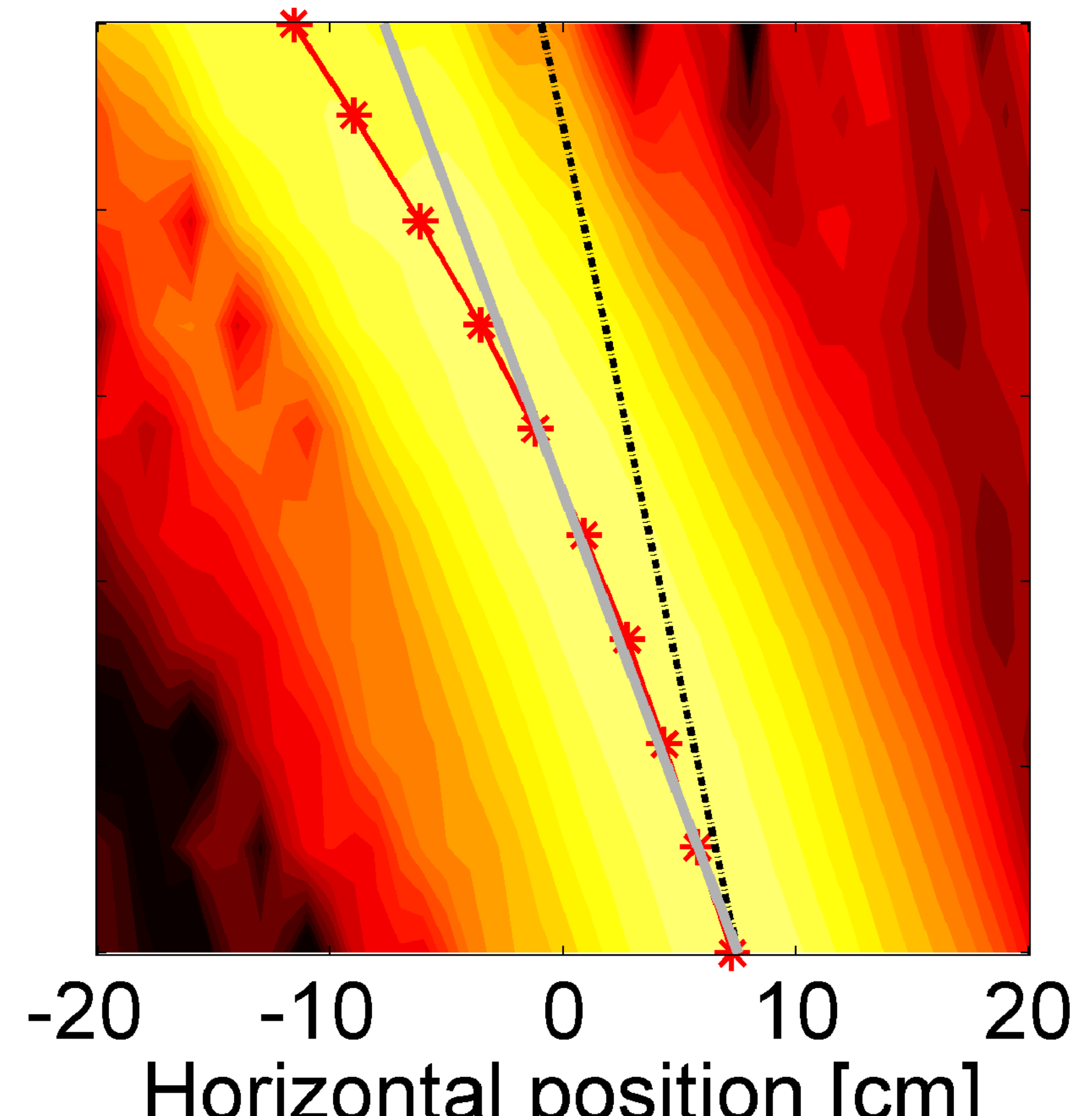
Horizontal position [cm]

(v)

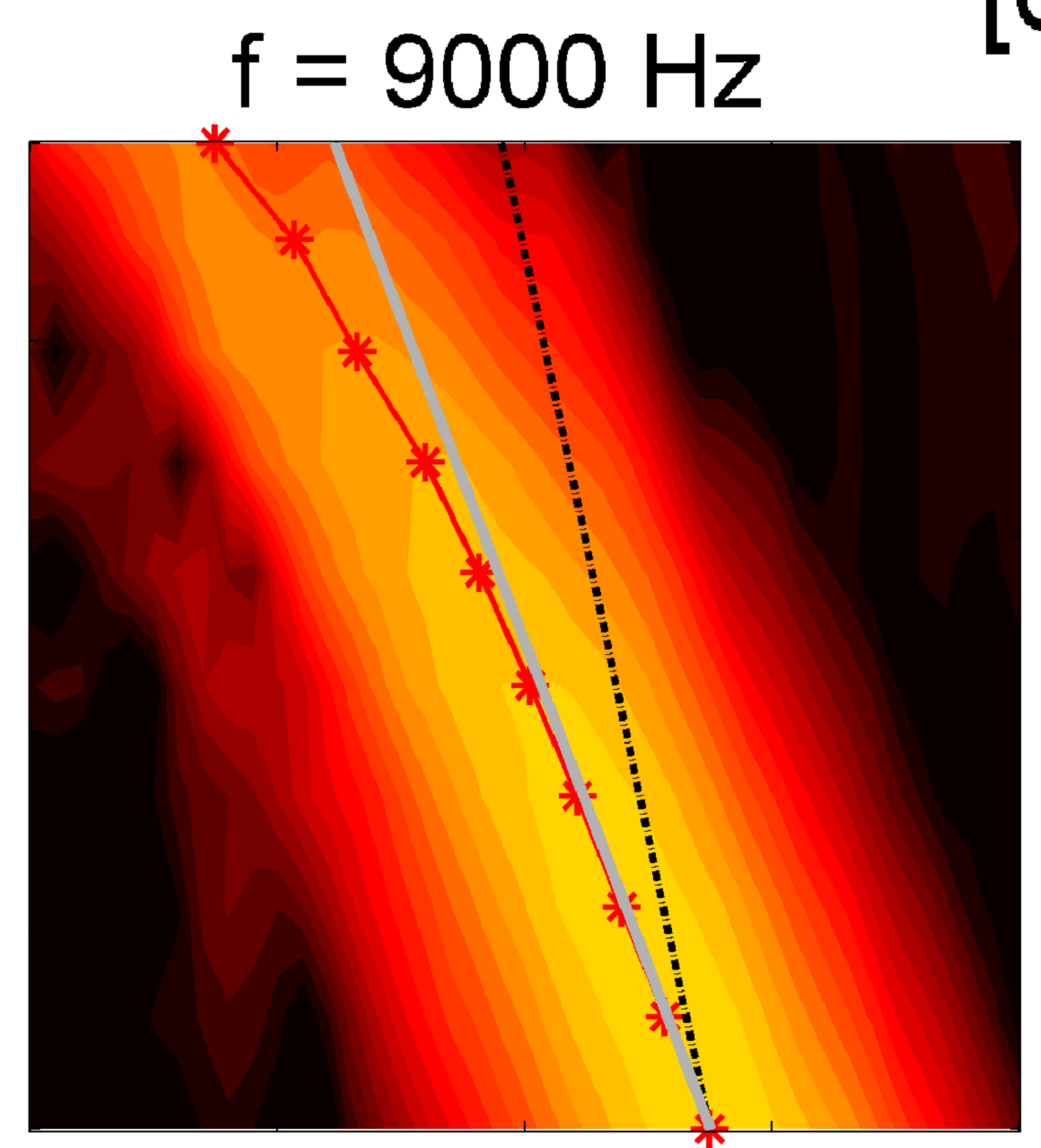

(iii)

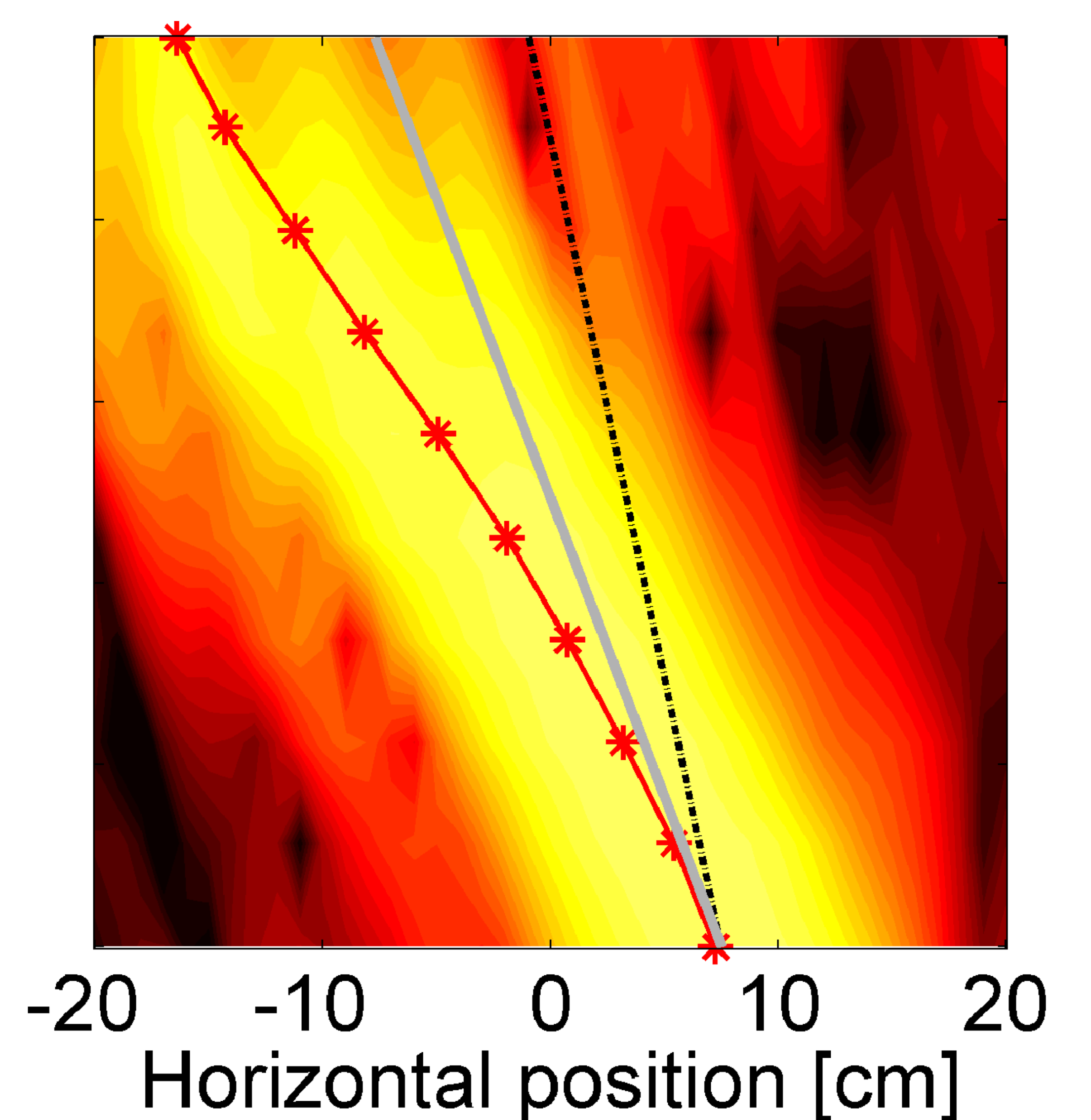

60

50

40 (iv)

(vi) 
